\title{
Design and Realization of Information Service System of Agricultural Expert Based on Wireless Mobile Communication Technology
}

\author{
Jianshe Zhao ${ }^{1, *}$, Wenyue $\mathrm{Li}^{1}$, Yong Yang ${ }^{2}$, Haili Meng ${ }^{1}$, and Wen Huang ${ }^{1}$ \\ ${ }^{1}$ Zhengzhou Vegetable Research Institute, Zhengzhou, 450015 P.R. China \\ js.zhao@hotmail.com \\ ${ }^{2}$ Zhengzhou S\&T Information Institute, Zhengzhou, 450015 P.R. China
}

\begin{abstract}
This paper elaborates the detail of designing of agriculture service platform system, which revolutionized agriculture information delivery in rural and underserved areas. Based on mobile wireless protocols, the platform allows mobile phones to capture and send data for an agricultural information and links farmers with agronomist for real-time decision support.
\end{abstract}

Keywords: SMS, Mobile communication, Service system, Agriculture.

\section{Introduction}

\subsection{Background of the Project}

The 2009 Investigation Report on the Development Status of Internet In Rural Areas of China" released by China Internet Information Center indicated that the rural netitzens in China has reached 106.81 million by the end of December 2009, the rural surfing population by mobile phone was about 71.89 million, it increased more than 30 million compared with 2008 and the annual growth rate reached $79.3 \%$, which is considerable higher than the overall growth rate of the rural netizens.

The report shows that less rural netitzens are using desktop computer as their visiting terminals, and the proportion drops to $68 \%$, while the proportion of surfing by mobile phone increases by nearly $20 \%$ and the utilization rate reaches $67.3 \%$; among the 277 million urban netizens, 160 million of them are using mobile phone to surf, and the utilization rate reaches $57.5 \%$ the mobile phone has become the main visiting terminal of the rural netizens, and also it is an importance motivity in activating the development of Internet in rural areas.

\subsection{Function and Innovation of the System}

The Short Message Service (SMS) is an important service of GSM; it can transfer data information with limited length through the GSM network. Depending on the SMS of GSM, this system can transfer inquiry instruction and inquiry result between

${ }^{*}$ Corresponding author.

D. Li, Y. Liu, and Y. Chen (Eds.): CCTA 2010, Part III, IFIP AICT 346, pp. 598-603, 2011.

(C) IFIP International Federation for Information Processing 2011 
the user and the system, and thus realize the mobile inquiry of the Information service system of vegetable experts. The paper analyzes the database access technology based on the SMPP, SGIP, and CMPP3.0 protocol. It puts forward a SMS agricultural information inquiry scheme, wherein the inquiry means to become the specific agricultural scientific \& technical Information into abstract data information, and provide guidance and solution on this basis. Through this method, the system can provide inquiry demand which is easy and fast for search. The innovative functions of the system include:

The data background supports fuzzy search and automatic classification \& matching. The character processing adopts the keywords take-out technology, a keywords database is set up to make automatic matching of the keywords according to the keywords; It can mark the various vegetable diseases and insect pests information and automatically classify and match them according to the keywords to look for the corresponding research experts.

Automatic and active update of the database: The system will create automatically a new question if there is no solution in the database and get answer from the experts. This change the database from passive update to active update, and the contents of the original database are enriched.

Dynamic statistic, analysis and processing decision of the vegetable diseases and insect pests: with the increasing visit to this system, the type, time and other rules of the questions raised by the farmers can be counted in a dynamic way, which can be utilized for making scientific choice in the prevention and treatment of the diseases and insect pests.

\section{System Design}

\subsection{Design Principle of the System}

This system utilizes the latest wireless network and software technology and follows the advanced development design standard, it mainly depends on the SMS system, the traditional experts E-mail and phone support system and WAP are the secondary means. The system pays equal attention to the social benefits and economic benefits. The following is the design principle of this system:

\section{Practical}

The overall design of the system fully considers the information system and experts resources of Zhengzhou Vegetable Research Institute, and the increasing importance of the mobile phone in rural area, also it considers the economic status of the rural farmers, this agricultural experts information service system is thus set up on the basis of the wireless mobile communication technology to provide expert solution for more farmers.

\section{Adaptable}

The system pays enough attention to the adaptability, and shall be easy for the various farmers; the system response shall be in accordance with the operation habit of the farmer, such as the fuzzy matching and dialect matching and so on. 


\section{Compatible}

During the research and development stage of the SMS application system, full consideration has been given to the CMPP protocol and SGIP protocol; this system is compatible with the SMS gateway interface protocol of China Mobile and China Unicom.

\section{Safe}

The system is completely safe in hardware, network, database and the power limit of data, application operation and user identification, the system has strong safety prevention measures and can stop the illegal access or destruction either in hardware, software, or service flow and so on.

\subsection{System Overview}

Farmers: They use handset to access the WBAEISS via the Wireless Base Station, they can send SMS to WBAEISS or they can also use on the WAP service of the WBAEISS. The farmer can ask different kinds of questions to the WBAEISS; include vegetable disease problem, pest problems and other agricultural related problems.

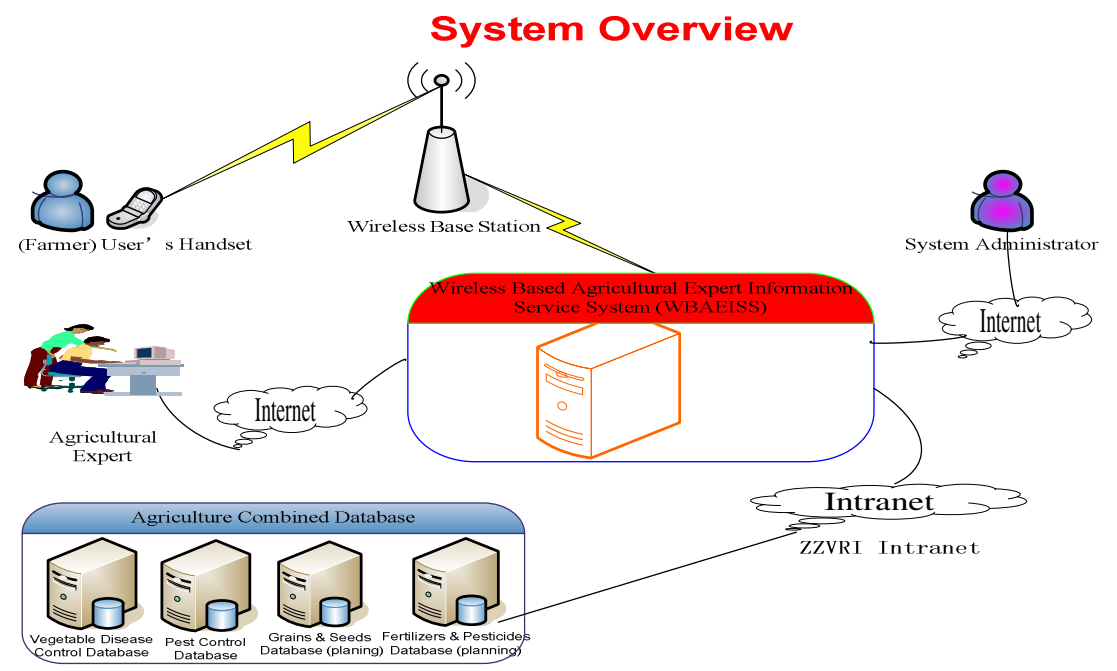

Fig. 1. The overview of the system shows the all participant: the actors, the network, the Database and Wireless Based Agricultural Expert Information Service System (WBAEISS).

Agricultural Experts: If there is no answer for some of farmers' questions in the current knowledge database of WBAEISS, the agricultural experts will receive e-mail or SMS from the system, they can use the system via web browser, such as IE, to add new answer to the knowledge database. The can also use the system to do some analysis/statistics work, base on the analysis/statistics results they make an expert decision to send some forecast on vegetable disease or pest control to farmers.

System Administrators: The system administrator use the web browser, such as IE, to do some maintenance work on WBAEISS, create new user, add new expert, back up database/log, and so on. 


\subsection{Design Architecture}

This chapter is focus on the design architecture. The design architecture of the system consists of four Layers and two kernel modules as the following System Architecture.

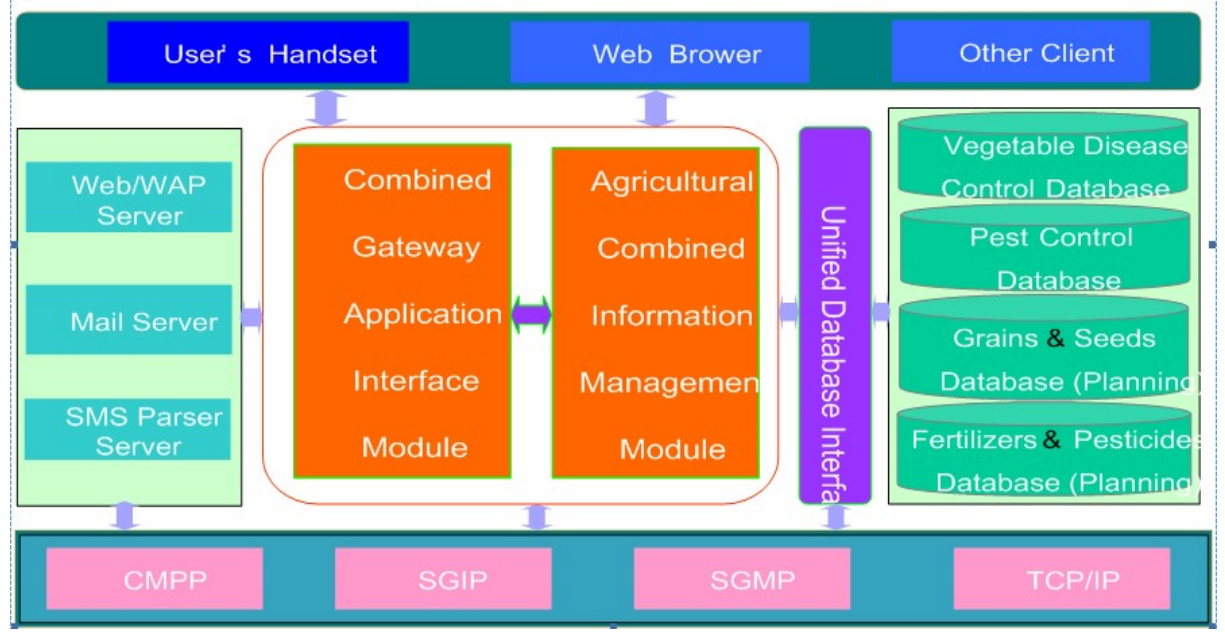

Fig. 2. System architecture

The Present Layer: This layer provides the GUI to the final users of the WBAEISS, including user's handset, web server and so on.

The Application Layer: This layer fulfils the WBAEISS system requirements, including the 2 kernel modules and 3 application servers.

Combined Gateway Application Interface Module: It is a unified interface to deal with the request from the different communication Medias: e-mail channel, SMS channel and web channel.

Agricultural Combined Information Management Module: Deal with the main business logic of the WBAEISS, including the request from the agricultural experts, the system administrator and the Combined Gateway Application Interface Module, and it also communications with the Unified Database interface.

Persistent Layer: This layer provides the unified database interface to storage, organization and retrieval of structured data, provide concurrency, data integrity and data sharing of the Agricultural combined database.

Communication Layer: This layer provides a module to support different protocols by different service providers, such as CMPP3.0 by China Mobile, SGIP by China Unicom, and SMGP by China Telcom... 


\section{System Functions Introduction}

This chapter introduces the main features of WBAEISS, as showed in the following figure, including 2 main function groups: agricultural combined information management and system management.

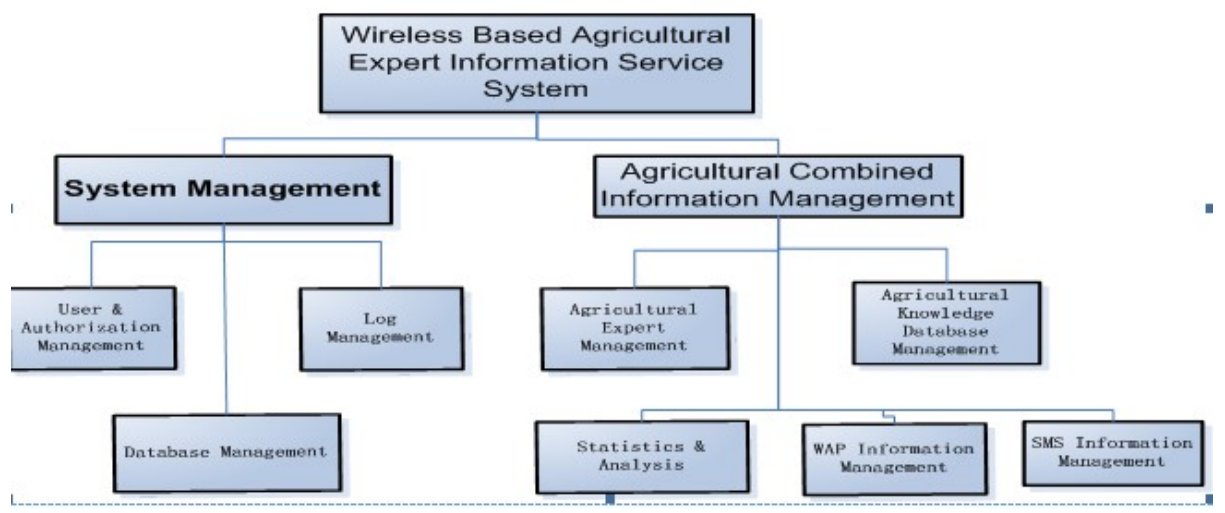

Fig. 3. System Function Structure Diagram

\subsection{Agricultural Combined Information Management}

Agricultural combined information management includes the following 5 features:

Agricultural Expert Management: The WBAEISS can add/delete different major agricultural experts, such as experts for pest, experts for vegetable disease and so on.

Agricultural Knowledge Database Management: The WBAEISS can gather new Agricultural Knowledge from the network, also from the answer to the farmers' question and so on. It also supports batch import and export. To make sure all the knowledge are correct, all the new answers/solutions must be checked by the agricultural experts.

Statistics and Analysis: The WBAEISS can help agricultural experts get statistics \& analysis results base on the questions of farmers by different time internal, day, month and year; by different question type, pest or vegetable disease... by different area, province, and city ... this module support customization.

SMS Information Management: The WBAEISS has a guide and workflow to help farmers to get the solution/answer they need, ie. The farmer sends "tomato, disease", the WBAEISS will response as

"tomato disease 1, symptom 1"

"tomato disease2, symptom 2"

...

And according to the selection by the farmer, the WBAEISS will give the right feedback that the farmer needed. The WBAEISS also supports some dialect, such as "fan qie" is tomato in the dialect of Henan province. 
WAP Information Management: The WBAEISS provides a WAP server for farmer to search their needed agricultural questions. The WBAEISS supports similar search and second search.

\subsection{System Management}

System management includes user \& authorization management, log management and database management.

User and Authorization Management: The WBAEISS supports add/edit/delete users; different type of users will have different authorization. Such as only System administrator can backup/resume the database.

Log Management: The WBAEISS can record the log of those delivery failure SMS and the system operation $\log$.

Database Management: The WBAEISS also provide the database backup/resume features, and WBAEISS also provide a feature to batch import/export the Database information.

\section{Conclusion and Future Work}

This service system supports more than one service providers, it can be accessed to several SPs, the Mobile, Unicom or Telecom, the only work is to add the configuration. It supports the various service items, and the module design of the system is convenient for the development of the new service. This system has been applied in the actual value-added SMS service, the connection to the gateways of China Mobile or Unicom shows good stability, which can satisfy the requirement of the system communication application Since the system was set up, it has been made known to the whole country through the nationwide vegetable business network, it receives thousands of SMS consultation every month, and it has become a common system for the breeding experts of vegetables, the vegetable growers and the distributors of agricultural commodities.

Depending on the Zhengzhou Scientific Data Sharing Project, it is planned to expand the contents of this system by adding the following: the database of scientific $\&$ technical achievements and the database of scientific \& technical literatures.

\section{References}

[1] Investigation Report on the Development Status of Interne In Rural Areas of China 2009 (in Chinese)

[2] Internet SMS gateway interface protocol 3.0 China Mobile communication Corporation (in Chinese)

[3] SMS gateway system interface protocol 1.2 China United communication Corporation (in Chinese)

[4] Xie, X.: Computer network. Electronic Industry Publishing House, Beijing (in Chinese)

[5] Pan, A.: The Low-down of Visual C++ Technology, 4th edn. Publishing House of Tsinghua University (in Chinese) 